ReVista de Matemática: Teoría y ApliCACiones 2017 24(1) : 97-113

CIMPA - UCR ISSN: 1409-2433 (PRINT), 2215-3373 (ONLINE)

\title{
ON A GENERALIZATION OF THE MAXIMUM ENTROPY THEOREM OF BURG
}

\section{SOBRE UNA GENERALIZACIÓN DE LA MÁXIMA ENTROPÍA TEOREMA DE BURG}

\author{
JOSÉ MARCANO* SABA INFANTE $^{\dagger} \quad$ LUIS SÁNCHEZ
}

Received: 20/Aug/2015; Revised: 25/Aug/2016;

Accepted: $18 /$ Oct/2016

\begin{abstract}
Revista de Matemática: Teoría y Aplicaciones is licensed under a Creative Commons Reconocimiento-NoComercial-Compartirigual 4.0 International License.

Creado a partir de la obra en http://www.revistas.ucr.ac.cr/index.php/matematica
\end{abstract}

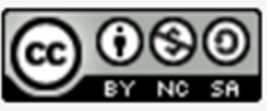

* Departamento de Matemáticas, Facultad de Ciencia y Tecnología, Universidad de Carabobo. Valencia, Venezuela. E-Mail: jmarcano@uc.edu.ve

${ }^{\dagger}$ Misma dirección que/Same address as: J. Marcano. E-Mail: sinfante64@gmail.com

${ }^{\ddagger}$ Departamento de Matemáticas, Facultad de Ciencias de la Educación, Universidad de Carabobo. Valencia, Venezuela. E-Mail: sluis@uc.edu.ve 


\begin{abstract}
In this article we introduce some matrix manipulations that allow us to obtain a version of the original Christoffel-Darboux formula, which is of interest in many applications of linear algebra. Using these developments matrix and Jensen's inequality, we obtain the main result of this proposal, which is the generalization of the maximum entropy theorem of Burg for multivariate processes.
\end{abstract}

Keywords: multivariate processes; maximum entropy theorem; Christoffel-Darboux formula.

\title{
Resumen
}

En este artículo se introducen algunas manipulaciones matriciales que nos permiten obtener una versión de la fórmula original de ChristoffelDarboux, la cual resulta de interés en muchas aplicaciones del álgebra lineal. Usando estos desarrollos matriciales y la desigualdad de Jensen, se obtiene el resultado principal de esta propuesta, que consiste en la generalización del teorema de entropía máxima de Burg para procesos multivariados.

Palabras clave: procesos multivariados; teorema de entropía máxima; fórmula de Christoffel-Darboux.

Mathematics Subject Classification: 94A17, 46E22.

\section{Introduction}

In 1975, Burg [2], in his doctoral thesis solved the following problem: given the first $p$ covariances $c_{0}, c_{1}, \ldots, c_{p}$ of a stationary stochastic process $X=$ $\left\{X_{n}\right\}_{n \in \mathbb{Z}}$, find the spectrum $f$ of the process $X$ which maximizes the functional

$$
\epsilon(g)=\frac{1}{2 \pi} \int_{0}^{2 \pi} \log g(t) d t
$$

subject to

$$
\frac{1}{2 \pi} \int_{0}^{2 \pi} g(t) e^{-i k t} d t=c_{k},|k| \leq p
$$

Since then many different authors have studied this problem, see for example [3], [8] and [10]. In 1993, Gabardo (cf. [7]), using the Krein functional (cf. [1]),

$$
\epsilon(g, \alpha)=\frac{1}{2 \pi} \int_{0}^{2 \pi} \log \left[g\left(e^{i t}\right)\right] \frac{1-|\alpha|^{2}}{\left|1-\alpha e^{-i t}\right|^{2}} d t
$$


generalized Burg's previous result. He showed that there exists a family of functions $\left\{w_{\alpha}\right\}$ ( $\alpha$ is a complex number with $\left.|\alpha| \leq 1\right)$ that satisfy (1) and the inequality

$$
\epsilon(g, \alpha) \leq \epsilon\left(w_{\alpha}, \alpha\right)
$$

for all spectra $g$ which satisfy (1).

The notion of entropy for multivariate time series was also studied by Burg in his doctorate thesis and later by other authors (cf. [2], [4] [9]). Gabardo [7] points out that Dym in [6] presents without proof a matrix version of the inequality (2), for the Wiener class.

Here we consider the Krein functional:

$$
\epsilon(\mathbf{W} ; \alpha)=\frac{1}{2 \pi} \int_{0}^{2 \pi} \frac{1-|\alpha|^{2}}{\left|1-\alpha e^{-i t}\right|^{2}} \log \operatorname{det} \mathbf{W}\left(e^{i t}\right) d t
$$

and show that there exists a family of matrix functions $\left\{\mathbf{W}_{\alpha}\right\}$ ( $\alpha$ is a complex number as before) that verifies the matrix version of (1), in such a way that the inequality $\epsilon(\mathbf{F}, \alpha) \leq \epsilon\left(\mathbf{W}_{\alpha}, \alpha\right)$ is valid for every matrix function $\mathbf{F}$ which is integrable on the unit circle satisfying the same restrictions as $\mathbf{W}_{\alpha}$.

The paper is organized as follows: in the second section we introduce some notations and preliminary results. In the third section we obtain a matrix version of the Christoffel-Darboux formula (cf. [5]). In the last section, we state the main result of the paper.

\section{Preliminaries}

In this section, we fix some notations and state a preliminary result given in [9].

Let $\mathbb{N}$ and $\mathbb{Z}$ stand for the set of natural and integer numbers. Let $\mathbb{C}$ denote the set of the complex numbers and $\mathbb{D}$ the open unit disk in the complex plane, i.e. $\mathbb{D}:=\{z \in \mathbb{C}:|z|<1\}$. The unit circle, the boundary of $\mathbb{D}$, will be denoted by $\mathbb{T}$. Given $n, m \in \mathbb{N}$, let $\mathbb{C}^{n \times m}$ be the set of all $n \times m$ matrices, $\mathbf{A}=\left\{A_{i, j}\right\}_{i=1, \ldots, n}^{j=1, \ldots, m}$ with $A_{i, j} \in \mathbb{C}, i=1, \ldots, n, j=1, \ldots, m$. If $m=1$, we write $\mathbb{C}^{n}$ instead of $\mathbb{C}^{n \times 1}$. If $\mathbf{A} \in \mathbb{C}^{n \times n}$ we write $\mathbf{A}>0$ if $u^{*} \mathbf{A} u>0$ for $u \in \mathbb{C}^{n}, u$ not null, and denote by $\mathbf{A}^{*} \in \mathbb{C}^{n \times n}$ the conjugated transpose of $A$. $u^{t}$ will denote the transpose of a vector $u$. Let

$$
\begin{aligned}
& L^{\infty}:=\left\{f: \mathbb{T} \rightarrow \mathbb{C}: f \text { Lebesgue measurable and ess } \sup _{\zeta \in \mathbb{T}}|f(\zeta)|<\infty\right\}, \\
& L^{1}:=\left\{f: \mathbb{T} \rightarrow \mathbb{C}: f \text { Lebesgue measurable and } \int_{0}^{2 \pi}\left|f\left(e^{i t}\right)\right| d t<\infty\right\},
\end{aligned}
$$


and $L^{2}=L^{2}(\mathbb{T})$ the space of functions $f, f: \mathbb{T} \longrightarrow \mathbb{C}$, measurable and square integrable (with respect to the Lebesgue measure, $d t$ on $\mathbb{T}$ ), with norm and inner product denoted by $\|\cdot\|$ and $\langle$,$\rangle , respectively. We write$

$$
\mathbf{L}^{\infty}\left(\mathbb{C}^{n \times m}\right):=\left\{\mathbf{F}: \mathbb{T} \rightarrow \mathbb{C}^{n \times m}: \mathbf{F}=\left\{F_{i, j}\right\}_{i=1, \ldots, n}^{j=1, \ldots, m} \text { with } F_{i, j} \in L^{\infty}\right\},
$$

and,

$$
\begin{aligned}
\mathbf{L}^{2}\left(\mathbb{C}^{n \times m}\right)= & \left\{\mathbf{F}: \mathbb{T} \rightarrow \mathbb{C}^{\mathbf{n} \times \mathbf{m}}: \mathbf{F}\right. \text { is measurable and } \\
& \left.\frac{1}{2 \pi} \int_{0}^{2 \pi}\left\langle\mathbf{F}\left(e^{i t}\right), \mathbf{F}\left(e^{i t}\right)\right\rangle_{\mathbb{C}^{n \times m}} d t<\infty\right\} .
\end{aligned}
$$

Given $\mathbf{F}, \mathbf{G} \in \mathbf{L}^{2}\left(\mathbb{C}^{n \times m}\right)$ the inner product is defined as

$$
\langle\mathbf{F}, \mathbf{G}\rangle_{L^{2}\left(\mathbb{C}^{n \times m}\right)}:=\frac{1}{2 \pi} \int_{0}^{2 \pi}\left\langle\mathbf{F}\left(e^{i t}\right), \mathbf{G}\left(e^{i t}\right)\right\rangle_{\mathbb{C}^{n \times m}} d t .
$$

If $m=1$ we write $L_{n}^{2}$ instead of $\mathbf{L}^{2}\left(\mathbb{C}^{n \times 1}\right)$. For $\mathbf{F} \in L^{2}\left(\mathbb{C}^{n \times m}\right)$ and $k \in \mathbb{Z}$, let the $k$-th Fourier coefficient of $\mathbf{F}$, denoted by $\widehat{\mathbf{F}}(k)$, be the matrix defined by $\widehat{\mathbf{F}}(k)=\left\{\widehat{F}_{i, j}(k)\right\}_{i=1, \ldots, n}^{j=1, \ldots, m}$ where $\widehat{F}_{i, j}(k)=\frac{1}{2 \pi} \int_{0}^{2 \pi} e^{-i k t} F_{i, j}\left(e^{i t}\right) d t$. If $\left\{\Omega_{i, j}\right\}_{i=1, \ldots, n}^{j=1, \ldots, m}$ is a set of finite measures defined on $\mathbb{T}$, we denote by $\Omega$ the matrix measure $\boldsymbol{\Omega}:=\left\{\Omega_{i, j}\right\}_{i=1, \ldots, n}^{j=1, \ldots, m}$. The $k$-th Fourier coefficient of $\boldsymbol{\Omega}, \widehat{\boldsymbol{\Omega}}(k)$ is the matrix $\widehat{\boldsymbol{\Omega}}(k)=\left\{\widehat{\Omega}_{i, j}(k)\right\}_{i=1, \ldots, n}^{j=1, \ldots, m}$ where $\widehat{\Omega}_{i, j}(k)=\frac{1}{2 \pi} \int_{0}^{2 \pi} e^{-i k t} d \Omega_{i, j}\left(e^{i t}\right)$. Define $e_{k}$ by $e_{k}(\zeta):=\zeta^{k}, \zeta \in \mathbb{T}, k \in \mathbb{Z}$ and recall that $\left\{e_{k}\right\}_{k \in \mathbb{Z}}$ is a complete orthonormal system for the Hilbert space $L^{2}$. If $\left\{E^{i}\right\}_{i=1, \ldots, n}$ is the canonical base of $\mathbb{C}^{n}$, then $\mathcal{B}:=\left\{e_{k} E^{i}: k \in \mathbb{Z}, i=1, \ldots, n\right\}$ is a complete orthonormal system of $L_{n}^{2}$. Also, if we define the matrix $\mathbf{E}^{i, j}=\left\{\delta_{i}(k) \delta_{j}(l)\right\}_{k=1, \ldots, n}^{l=1, \ldots, m}$ then $\left\{e_{k} \mathbf{E}^{i, j}: k \in \mathbb{Z}, i=1, \ldots, n, j=1, \ldots, m\right\}$ is a complete orthonormal system of $\mathbf{L}^{2}\left(\mathbb{C}^{n \times m}\right)$. Thus, if $\mathbf{F} \in L^{2}\left(\mathbb{C}^{n \times m}\right)$ then $\mathbf{F}=\sum_{k \in \mathbb{Z}} e_{k} \widehat{\mathbf{F}}(k)$. Furthermore, $\widehat{\mathbf{F}}(k)=\sum_{i=1}^{n} \sum_{j=1}^{m}\left\langle\mathbf{F}, e_{k} \mathbf{E}^{i, j}\right\rangle_{\mathbf{L}^{2}\left(\mathbb{C}^{n \times m}\right)} \mathbf{E}^{i, j}, k \in \mathbb{Z}$. In particular, if $f \in L_{n}^{2}$ then $f=\sum_{k \in \mathbb{Z}} e_{k} \widehat{f}(k)$ with $\widehat{f}(k)=\sum_{i=1}^{n}\left\langle f, e_{k} E^{i}\right\rangle_{L^{2}\left(\mathbb{C}^{n}\right)} E^{i}, k \in \mathbb{Z}$. Also, $H^{\infty}(\mathbb{D})$ denotes the set of analytic functions $f$ on $\mathbb{D}$, such that its norm $\|f\|_{\infty}=\sup _{z \in \mathbb{D}}|f(z)|$ is finite and $\mathbf{H}_{n \times m}^{\infty}(\mathbb{D})$ denotes the space of analytic matrices whose inputs are in $H^{\infty}(\mathbb{D})$ and $H^{\infty}$ the space $H^{\infty}:=H^{\infty}(\mathbb{T})=\{f \in$ $L^{\infty}(\mathbb{T}): \widehat{f}(n)=0$ if $\left.n<0\right\}$.

A sequence of $q \times q$ complex matrices $\left\{\mathbf{R}_{k}\right\}_{k=-p}^{p} \subset \mathbb{C}^{q \times q}$, is said to be strictly positive definite if, and only if,

$$
\sum_{n=0}^{p} \sum_{m=0}^{p}\left\langle\mathbf{R}_{m-n} f_{n}, f_{m}\right\rangle_{\mathbb{C}^{q}}>0
$$

for each $\left\{f_{k}\right\}_{k=0}^{p} \subset \mathbb{C}^{q}$ not null. 
We can prove that if the sequence $\left\{\mathbf{R}_{k}\right\}_{k=-p}^{p}$ is strictly positive definite, then $\mathbf{R}_{k}=\mathbf{R}_{-k}^{*}$ for $k=1, \cdots, p$. Suppose that the sequence of matrices $\left\{\mathbf{R}_{k}\right\}_{k=-p}^{p}$ verifies (3) with $\mathbf{R}_{0}=\mathbf{I}_{q \times q}$. Let $\mathcal{E}_{p}=\mathcal{E}_{p}\left(\mathbb{C}^{q}\right)$ be the manifold spanned by $\left\{e_{k} E^{j}\right\}_{k=0, \ldots, p}^{j=1, \ldots, q}$, this is, the set of all vectors whose components are trigonometric polynomials on $\mathbb{T}$, of degree at most $p$. Clearly, if we define the inner product in $\mathcal{E}_{p}$ by

$$
\left\langle\sum_{n=0}^{p} e_{n} f_{n}, \sum_{m=0}^{p} e_{m} g_{m}\right\rangle_{p}=\sum_{n=0}^{p} \sum_{m=0}^{p}\left\langle\mathbf{R}_{m-n} f_{n}, g_{m}\right\rangle_{\mathbb{C}^{q}},\left\{f_{n}\right\}_{n=0}^{p},\left\{g_{n}\right\}_{n=0}^{p} \in \mathbb{C}^{q}
$$

then $\left(\mathcal{E}_{p},\langle,\rangle_{p}\right)$ is a Hilbert space of dimension $(p+1) q$.

Let $\Gamma_{p}:\left(\mathcal{E}_{p},\langle, .,\rangle_{p}\right) \rightarrow\left(\mathcal{E}_{p},\langle, .,\rangle_{L_{q}^{2}}\right)$ be the linear operator defined by the relation

$$
\left\langle\Gamma_{p} f, g\right\rangle_{L_{q}^{2}}:=\langle f, g\rangle_{p}
$$

Let us notice that

$$
\Gamma_{p}(f)=\sum_{k=0}^{p} e_{k} \sum_{s=0}^{p} \mathbf{R}_{k-s} \widehat{f}(s)
$$

Let $\mathcal{D}_{p}=\operatorname{Span}\left\{e_{k} E^{s}\right\}_{k=0,1, \ldots, p-1}^{s=1, \ldots, q}, \mathcal{R}_{p}=\operatorname{Span}\left\{e_{k} E^{s}\right\}_{k=1, \ldots, p}^{s=1, \ldots, q}$ be the subspaces of $\mathcal{E}_{p}$ and let $V_{p}: \mathcal{D}_{p} \longrightarrow \mathcal{R}_{p}$ be defined as the linear extension of $V_{p}\left(e_{k} E^{s}\right)=e_{k+1} E^{s}$. Then,

(a) $V_{p}$ is an isometry acting on the space $\left(\mathcal{E}_{p},\langle,\rangle_{p}\right)$.

(b) The operator $\Gamma_{p}$ is an isomorphism.

(c) The defect subspaces of the isometry $V_{p}, \mathcal{N}_{p}=\mathcal{E}_{p} \ominus \mathcal{D}_{p}$ and $\mathcal{M}_{p}=$ $\mathcal{E}_{p} \ominus \mathcal{R}_{p}$ have dimension $q$ and are given by $\mathcal{N}_{p}=\operatorname{Span}\left\{\Gamma_{p}^{-1} e_{p} E^{s}\right\}_{s=1}^{q}$ and $\mathcal{M}_{p}=\operatorname{Span}\left\{\Gamma_{p}^{-1} e_{0} E^{s}\right\}_{s=1}^{q}$ respectively.

Remark 1 We remark that $\left(\mathcal{E}_{p-1},\langle,\rangle_{L_{q}^{2}}\right)$ is a subspace of $\left(\mathcal{E}_{p},\langle,\rangle_{L_{q}^{2}}\right)$ and from (6) it is easy to see

$$
\left.P_{\mathcal{E}_{p-1}}^{\mathcal{E}_{p}} \Gamma_{p}\right|_{\mathcal{E}_{p-1}}=\Gamma_{p-1}
$$

where $\left.\Gamma_{p}\right|_{A}$ is the restriction of the operator $\Gamma_{p}$ to the set $A, A \subset \mathcal{E}_{p}$ and the operator $P_{A}^{B}$ is the orthogonal projection of $B$ over $A$. Thus, if $x, y \in \mathcal{E}_{p-1}$

$$
\langle x, y\rangle_{p}=\left\langle\Gamma_{p} x, y\right\rangle_{L_{q}^{2}}=\left\langle\Gamma_{p-1} x, y\right\rangle_{L_{q}^{2}}=\langle x, y\rangle_{p-1}
$$


Analogously, if $1 \leq j<k \leq p$ then the identity $I_{j, k}:\left(\mathcal{E}_{j},\langle,\rangle_{j}\right) \rightarrow\left(\mathcal{E}_{k},\langle,\rangle_{k}\right)$ is an isometry. For $k=p$ and $j=p-1$ we set $I_{p-1, p}:=J$. Therefore, we conclude that if $x, y$ are orthogonal in $\mathcal{E}_{p-1}$, then $x$ and $y$ are orthogonal in $\mathcal{E}_{p}$. With the above notation before the remark, it is easy to check that

- $\mathcal{D}_{p}=J\left(\mathcal{D}_{p-1} \oplus \mathcal{N}_{p-1}\right)=J\left(\mathcal{R}_{p-1} \oplus \mathcal{M}_{p-1}\right)=J\left(V_{p} \mathcal{D}_{p-1} \oplus \mathcal{M}_{p-1}\right)$,

- $\mathcal{R}_{p}=V_{p} \mathcal{D}_{p}=J\left(\mathcal{R}_{p-1} \oplus V_{p} \mathcal{N}_{p-1}\right)$.

Remark 2 If $\left\{m_{p}^{k}\right\}_{k=1}^{q}$ and $\left\{n_{p}^{k}\right\}_{k=1}^{q}$ are the orthonormal vectors in $\left(\mathcal{E}_{p},\langle,\rangle_{p}\right)$ obtained by applying the Gram-Schmidt method to $\left\{\Gamma_{p}^{-1} e_{0} E^{k}\right\}_{k=1}^{q}$ and $\left\{\Gamma_{p}^{-1} e_{p} E^{k}\right\}_{k=1}^{q}$ respectively, then

$$
\begin{aligned}
& m_{p}^{k}=\sum_{j=1}^{q} A_{p}(j, k) \Gamma_{p}^{-1} e_{0} E^{j}, k=1, \cdots, q \\
& n_{p}^{k}=\sum_{j=1}^{q} B_{p}(j, k) \Gamma_{p}^{-1} e_{p} E^{j}, k=1, \cdots, q .
\end{aligned}
$$

Let $n_{p}^{k}=\left(n_{p}^{k}(1) \cdots n_{p}^{k}(q)\right)^{t}$ and $m_{p}^{k}=\left(m_{p}^{k}(1) \cdots m_{p}^{k}(q)\right)^{t}$. Let us consider $\mathbf{M}_{p}(\zeta), \mathbf{N}_{p}(\zeta), \zeta \in \mathbb{T}$, the matrices defined by

$$
\mathbf{N}_{p}=\left\{n_{p}^{j}(i)\right\}_{i, j=1, \ldots, q} \text { and } \mathbf{M}_{p}=\left\{m_{p}^{j}(i)\right\}_{i, j=1, \ldots, q}
$$

and

$$
\mathbf{A}_{p}=\left\{A_{p}(i, j)\right\}_{i, j=1, \ldots, q} \text { and } \mathbf{B}_{p}=\left\{B_{p}(i, j)\right\}_{i, j=1, \ldots, q}
$$

where the coefficients $A_{p}(i, j)$ and $B_{p}(i, j) ; i, j=1, \ldots, q$ are given by (7). From [9] we know that all the zeros of $\operatorname{det} \mathbf{N}_{p}$ and $\operatorname{det} \mathbf{M}_{p}$ lie in the open unit disk $\mathbb{D}$ and in the exterior of the closed unit disk, respectively.

\section{The Christoffel-Darboux formula}

The main result of this section is that $\mathcal{E}_{p}$ is a reproducing kernel space. As usual, we denote by $\mathcal{L}\left(\mathbb{C}^{q}\right)$, the set of all bounded linear operator acting on $\mathbb{C}^{q}$.

Definition 1 We say that the Hilbert space $\mathcal{H}$ of functions $f: X \rightarrow \mathbb{C}^{q}$, has a reproducing kernel if there exists an application

$$
K: X \times X \rightarrow \mathcal{L}\left(\mathbb{C}^{q}\right)
$$

satisfying the following properties 
(a) For each $x \in X, \xi \in \mathbb{C}^{q}$ we have that $K_{x} \xi \in \mathcal{H}$, where $K_{x}(y)=K(x, y)$ and $\left\langle K_{x} \xi, K_{y} \eta\right\rangle_{\mathcal{H}}=\langle K(x, y) \xi, \eta\rangle_{\mathbb{C}^{q}}$.

(b) For each $f \in \mathcal{H}, \xi \in \mathbb{C}^{q}, y \in X$ we have $\langle f(y), \xi\rangle_{\mathbb{C}^{q}}=\left\langle f, K_{y} \xi\right\rangle_{\mathcal{H}}$.

The next proposition shows that $\mathcal{E}_{p}\left(\mathbb{C}^{q}\right)$ can be considered a reproducing kernel space.

Proposition 1 For each $z \in \mathbb{C}$, let $T_{p}^{z}: \mathbb{T} \rightarrow \mathcal{L}\left(\mathbb{C}^{q}\right)$ be the function defined by $\left(T_{p}^{z}\right) x=\Gamma_{p}^{-1}\left(\sum_{k=0}^{p} \bar{z}^{k} e_{k} x\right)$. Then

$$
\langle P(z), \xi\rangle_{\mathbb{C}^{q}}=\left\langle P,\left(T_{p}^{z}\right) \xi\right\rangle_{p} ; \xi \in \mathbb{C}^{q}, P \in \mathcal{E}_{p} .
$$

Furthermore,

$$
\left(T_{p}^{z}\right) \xi=\sum_{k=0}^{p} \sum_{i=1}^{q}\left\langle\left(T_{p}^{z}\right) \xi, n_{k}^{i}\right\rangle_{p} n_{k}^{i}, \xi \in \mathbb{C}^{q}
$$

where $n_{0}^{i}=e_{0} E^{i}$ and $n_{k}^{i}, k=1, \ldots, p$ and $i=1, \ldots, q$ are as in (7).

Proof: Note that $\left(T_{p}^{z}\right) x \in \mathcal{E}_{p}$ for each $x \in \mathbb{C}^{q}$. Let $P \in \mathcal{E}_{p}$. Thus,

$$
\begin{aligned}
\left\langle P,\left(T_{p}^{z}\right) x\right\rangle_{p} & =\left\langle P, \Gamma_{p}^{-1}\left(\sum_{k=0}^{p} \bar{z}^{k} e_{k} x\right)\right\rangle_{p}=\left\langle P, \sum_{k=0}^{p} \bar{z}^{k} e_{k} x\right\rangle_{L_{q}^{2}} \\
& =\frac{1}{2 \pi} \int_{0}^{2 \pi}\left\langle P, \sum_{k=0}^{p} \bar{z}^{k} e^{i t} x\right\rangle_{\mathbb{C}^{q}} d t \\
& =\sum_{k=0}^{p} z^{k} \frac{1}{2 \pi} \int_{0}^{2 \pi}\langle P, x\rangle_{\mathbb{C}^{q}} e^{-i k t} d t \\
& =\sum_{l=0}^{p} \sum_{k=0}^{p} z^{k}\langle\widehat{P}(l), x\rangle_{\mathbb{C}^{q}} \frac{1}{2 \pi} \int_{0}^{2 \pi} e^{-i(k-l) t} d t \\
& =\sum_{k=0}^{p} z^{k}\langle\widehat{P}(k), x\rangle_{\mathbb{C}^{q}}=\langle P(z), x\rangle_{\mathbb{C}^{q} .}
\end{aligned}
$$

Finally, that $\left(T_{p}^{z}\right) x=\sum_{k=0}^{p} \sum_{i=1}^{q}\left\langle\left(T_{p}^{z}\right) x, n_{k}^{i}\right\rangle_{p} n_{k}^{i}$ follows now the fact that $\left\{n_{k}^{i}\right\}_{k=0,1, \ldots, p}^{i=1, \ldots, q}$ is a orthonormal system of $\mathcal{E}_{p}$.

From this proposition, we obtain the reproducing kernel $K(z, \xi)=T_{p}^{\xi}(z)$.

As a consequence of proposition 1 , we obtain a version of the ChristoffelDarboux formula (see [5]). 
Theorem 1 (The Christoffel-Darboux formula) If $z \bar{\xi} \neq 1$ then

$$
\mathbf{T}_{p}^{z}(\xi)=\frac{\mathbf{M}_{p}(\xi) \mathbf{M}_{p}^{*}(z)-\xi \mathbf{N}_{p}(\xi) \bar{z} \mathbf{N}_{p}^{*}(z)}{1-\xi \bar{z}}
$$

Proof: Let $Q \in \mathcal{E}_{p-1}$ and $z, \xi \in \mathbb{D}$. From the preceding proposition, remark 1 and the fact that $V_{p+1}$ is an isometry, we obtain that

$$
\begin{aligned}
0 & =\left\langle\left(e_{1}-z\right) Q,\left(T_{p}^{z}\right) x\right\rangle_{p} \\
& =\left\langle e_{1} Q,\left(T_{p}^{z}\right) x\right\rangle_{p+1}-\left\langle e_{1} Q, e_{1} \bar{z}\left(T_{p}^{z}\right) x\right\rangle_{p+1} \\
& =\left\langle e_{1} Q,\left(1-\bar{z} e_{1}\right)\left(T_{p}^{z}\right) x\right\rangle_{p+1} .
\end{aligned}
$$

Using the fact that $\mathcal{R}_{p}=\operatorname{Span}\left\{e_{1} Q: Q \in \mathcal{E}_{p-1}\right\}$ we obtain $\left(1-\bar{z} e_{1}\right)\left(T_{p}^{z}\right) x \in \mathcal{E}_{p+1} \ominus \mathcal{R}_{p}$ the orthogonal complement of the subspace $\mathcal{R}_{p}$ with respect to the $q(p+1)$-dimensional space $\mathcal{E}_{p+1}$. On the other hand, $\left\{e_{1} n_{p}^{s}\right\}_{s=1}^{q}$ are orthonormal and $\left\{\left(T_{p}^{0}\right) E^{s}\right\}_{s=1}^{q}=\left\{\Gamma_{p}^{-1}\left(e_{0} E^{s}\right)\right\}_{s=1}^{q}$ are linearly independent. From (8) we know that the orthonormal system $\left\{m_{p}^{l}\right\}_{l=1}^{q}$ is obtained by applying the Gram-Schmidt process to $\left\{\left(T_{p}^{0}\right) E^{s}\right\}_{s=1}^{q}$. Also,

$$
\begin{aligned}
\left\langle e_{1} n_{p}^{k}, m_{p}^{l}\right\rangle_{p+1} & =\left\langle e_{1} n_{p}^{k}, \sum_{j=1}^{q} A_{p}(j, l) \Gamma_{p}^{-1} e_{0} E^{j}\right\rangle_{p+1} \\
& =\sum_{j=1}^{q} \overline{A_{p}(j, l)}\left\langle e_{1} n_{p}^{k}, \Gamma_{p}^{-1} e_{0} E^{j}\right\rangle_{p+1} \\
& =\sum_{j=1}^{q} \overline{A_{p}(j, l)}\left\langle e_{1} n_{p}^{k},\left(T_{p}^{0}\right) E^{j}\right\rangle_{p+1}=0 .
\end{aligned}
$$

Clearly, $\left\{e_{1} n_{p}^{s}\right\}_{s=1}^{q}$ and $\left\{m_{p}^{l}\right\}_{s=1}^{q}$ belong to $\mathcal{E}_{p+1} \ominus \mathcal{R}_{p}$ since $Q \in \mathcal{E}_{p-1}$,

$$
\left\langle e_{1} n_{p}^{k}, e_{1} Q\right\rangle_{p+1}=\left\langle n_{p}^{k}, Q\right\rangle_{p+1}=0
$$

and

$$
\begin{aligned}
\left\langle m_{p}^{k}, e_{1} Q\right\rangle_{p+1} & =\left\langle\sum_{j=1}^{q} A_{p}(j, k) \Gamma_{p}^{-1} e_{0} E^{j}, e_{1} Q\right\rangle_{p+1} \\
& =\sum_{j=1}^{q} A_{p}(j, k)\left\langle\left(T_{p}^{0}\right) E^{j}, e_{1} Q\right\rangle_{p+1}=0 .
\end{aligned}
$$


Thus, $\left\{e_{1} n_{p}^{s}\right\}_{s=1}^{q}$ and $\left\{m_{p}^{l}\right\}_{s=1}^{q}$ both orthonormal systems, generate the 2qdimensional space $\mathcal{E}_{p+1} \ominus \mathcal{R}_{p}$. Therefore,

$$
\left(1-\bar{z} e_{1}\right)\left(T_{p}^{z}\right) x=\sum_{s=1}^{q} a_{s} m_{p}^{s}+\sum_{s=1}^{q} b_{s} e_{1} n_{p}^{s} .
$$

As a consequence of the previous proposition,

$$
\left(T_{p}^{z}\right) x=\sum_{k=0}^{p} \sum_{i=1}^{q}\left\langle\left(T_{p}^{z}\right) x, n_{k}^{i}\right\rangle_{p} n_{k}^{i}
$$

Hence,

$$
-\bar{z} e_{1}\left(T_{p}^{z}\right) x=\sum_{i=1}^{q}\left(-\bar{z}\left\langle\left(T_{p}^{z}\right) x, n_{k}^{i}\right\rangle_{p}\right) e_{1} n_{p}^{i}+\sum_{k=0}^{p-1} \sum_{i=1}^{q}\left(-\bar{z}\left\langle\left(T_{p}^{z}\right) x, n_{k}^{i}\right\rangle_{p}\right) e_{1} n_{k}^{i},
$$

and,

$$
\begin{aligned}
\left(1-z e_{1}\right)\left(T_{p}^{z}\right) x & =\sum_{i=1}^{q}\left(-\bar{z}\left\langle\left(T_{p}^{z}\right) x, n_{k}^{i}\right\rangle_{p}\right) e_{1} n_{p}^{i} \\
& +\left(\left(T_{p}^{z}\right) x+\sum_{k=0}^{p-1} \sum_{i=1}^{q}\left(-\bar{z}\left\langle\left(T_{p}^{z}\right) x, n_{k}^{i}\right\rangle_{p}\right) e_{1} n_{k}^{i}\right) .
\end{aligned}
$$

From (13) and (14),

$$
\begin{aligned}
\sum_{s=1}^{q} b_{s}\left\langle e_{1} n_{p}^{s}, e_{p+1} E^{r}\right\rangle_{L_{q}^{2}} & =\left\langle\left(1-\bar{z} e_{1}\right)\left(T_{p}^{z}\right) x, e_{p+1} E^{r}\right\rangle_{L_{q}^{2}} \\
& =\sum_{s=1}^{q}\left(-\bar{z}\left\langle x, n_{p}^{s}(z)\right\rangle_{\mathbb{C}^{q}}\right)\left\langle e_{1} n_{p}^{s}, e_{p+1} E^{r}\right\rangle_{L_{q}^{2}}
\end{aligned}
$$

Then, $b_{s}=-\bar{z}\left\langle x, n_{p}^{s}(z)\right\rangle_{\mathbb{C} q}, s=1, \ldots, q$.

From (13),

$$
\left\langle\left(1-z e_{1}\right)\left(T_{p}^{z}\right) x, m_{p}^{j}\right\rangle_{p+1}=\sum_{s=1}^{q} a_{s}\left\langle m_{p}^{s}, m_{p}^{j}\right\rangle_{p+1} .
$$

With the same procedure used in (12) we prove $\left\langle e_{1}\left(T_{p}^{z}\right) x, m_{p}^{j}\right\rangle_{p+1}=0$, therefore,

$$
a_{s}=\left\langle\left(T_{p}^{z}\right) x, m_{p}^{s}(z)\right\rangle_{p+1}=\left\langle x, m_{p}^{s}(z)\right\rangle_{\mathbb{C}^{q}}, s=1, \ldots, q
$$

Rev.Mate.Teor.Aplic. (ISSN print: 1409-2433; online: 2215-3373) Vol. 24(1): 97-113, January 2017 
and,

$$
\begin{aligned}
(1-\bar{z} \xi)\left(T_{p}^{z}\right)(\xi) x= & \sum_{s=1}^{q}\left\langle x, \mathbf{M}_{p}(z) E^{s}\right\rangle_{\mathbb{C}^{q}} \mathbf{M}_{p}(\xi) E^{s} \\
& +\sum_{s=1}^{q}(-\bar{z})\left\langle x, \mathbf{N}_{p}(z) E^{s}\right\rangle_{\mathbb{C}^{q}} \xi \mathbf{N}_{p}(\xi) E^{s}
\end{aligned}
$$

Therefore,

$$
\begin{aligned}
(1-\bar{z} \xi)\left\langle\left(T_{p}^{z}\right)(\xi) E^{j}, E^{k}\right\rangle_{\mathbb{C}^{q}} & =\sum_{s=1}^{q}\left\langle E^{j}, \mathbf{M}_{p}(z) E^{s}\right\rangle_{\mathbb{C}^{q}}\left\langle\mathbf{M}_{p}(\xi) E^{s}, E^{k}\right\rangle_{\mathbb{C}^{q}} \\
& +\sum_{s=1}^{q}(-\bar{z})\left\langle E^{j}, \mathbf{N}_{p}(z) E^{s}\right\rangle_{\mathbb{C}^{q}} \xi\left\langle\mathbf{N}_{p}(\xi) E^{s}, E^{k}\right\rangle_{\mathbb{C}^{q}},
\end{aligned}
$$

which yields the desired result.

\section{The covariance extension problem and the Krein entropy}

In 1980, Arov and Krein (cf. [1]) considered the entropy functional

$$
\epsilon(\mathbf{W} ; \alpha)=\frac{1}{2 \pi} \int_{0}^{2 \pi} \frac{1-|\alpha|^{2}}{\left|1-\alpha e^{-i t}\right|^{2}} \log \operatorname{det} \mathbf{W}\left(e^{i t}\right) d t, \alpha \in \mathbb{C} .
$$

The main objective of this paper is to show that there exists a family of matrix functions $\left\{\mathbf{W}_{\alpha}\right\}_{\{\alpha \in \mathbb{C}:|\alpha| \leq 1\}}$ verifying $\widehat{\mathbf{W}}(k)=\mathbf{R}_{k}, k=0,1, \ldots, p$, (where the matrices $\mathbf{R}_{k}$ are given) in such way that the following inequality $\epsilon(\mathbf{F}, \alpha) \leq$ $\epsilon\left(\mathbf{W}_{\alpha}, \alpha\right)$ is valid for all matrix functions $\mathbf{F}$ which are integrable on the unitary circle with $\widehat{\mathbf{F}}(k)=\mathbf{R}_{k}, k=0,1, \ldots, p$.

For this, we use the parametrization stated in [9] of the following problem: given $q \times q$ covariance matrices of the multivariate stochastic processes $\mathbf{R}_{0}, \mathbf{R}_{1}, \cdots, \mathbf{R}_{p}$, with $\mathbf{R}_{0}=\mathbf{I}$, we want to determine all $q \times q$ positive densities $\mathbf{F}$ on $\mathbb{T}$ so that

$$
\frac{1}{2 \pi} \int_{0}^{2 \pi} e^{-i k t} \mathbf{F}\left(e^{i k t}\right) d t=\mathbf{R}_{k}, k=0,1, \cdots, p .
$$

We show that there exists a one to one correspondence between the open unit ball of the space $\mathbf{H}_{q \times q}^{\infty}(\mathbb{D})$ defined as usual and the set of all the solutions of 
the problem. Under this correspondence we characterize the set of all measures which are absolutely continuous with respect to the Lebesgue matrix measure on $\mathbb{T}$. The spectral matrix densities are given by

$$
\mathbf{F}_{\mathbf{H}}(\zeta)=\left(\mathbf{N}_{p}^{*}\right)^{-1}\left[\left(\mathbf{N}_{p}^{-1} \mathbf{M}_{p}-\zeta \mathbf{H}\right)^{*}\right]^{-1}\left(\mathbf{I}_{q \times q}-\mathbf{H}^{*} \mathbf{H}\right)\left[\mathbf{N}_{p}^{-1} \mathbf{M}_{p}-\zeta \mathbf{H}\right]^{-1} \mathbf{N}_{p}^{-1}
$$

where $\mathbf{M}_{p}$ and $\mathbf{N}_{p}$ are as in (8) and $\mathbf{H} \in \mathbf{H}_{q \times q}^{\infty}(\mathbb{D})$ with $\mathbf{H}^{*} \mathbf{H}<\mathbf{I}_{q \times q}$ a.e. on $\mathbb{T}$.

First, we state the scalar covariance extension problem (see [10]): Given $p \in \mathbb{N}$, and $c_{0}, c_{1}, \cdots, c_{p}$, complex numbers with $c_{0}>0$ and $c_{-k}=\overline{c_{k}}, k=$ $1, \ldots, p$ find a positive density $f$ on $\mathbb{T}$ so that

$$
\frac{1}{2 \pi} \int_{0}^{2 \pi} e^{-i k t} f\left(e^{i k t}\right) d t=c_{k}, k=-p, \cdots, p .
$$

This problem has solution if and only if the finite sequence $\left\{c_{k}\right\}_{k=-p}^{p}$ is strictly positive definite and moreover, given $H \in H^{\infty}(\mathbb{D})$ such that $\|H\|_{\infty} \leq 1$, the set $\{\zeta \in \mathbb{T}:|H(\zeta)|=1\}$ has Lebesgue measure zero and $1 /\left(m_{p}-\zeta H n_{p}\right) \in$ $L^{2}$, the description of all the desired $f$ is given by

$$
f_{H}(\zeta)=\frac{1}{\left|m_{p}(\zeta)\right|^{2}} \operatorname{Re}\left[\frac{m_{p}(\zeta)+\zeta H(\zeta) n_{p}(\zeta)}{m_{p}(\zeta)-\zeta H(\zeta) n_{p}(\zeta)}\right], \zeta \in \mathbb{T}
$$

where $m_{p}$ and $n_{p}$ are as in (7) when $q=1$.

Consider the function $w_{\alpha}$ defined by

$$
w_{\alpha}\left(e^{i \theta}\right)=\frac{\left(1-|\alpha|^{2}\right)\left\|T_{p}^{\alpha}\right\|_{p}^{2}}{\left|1-\alpha e^{-i \theta}\right|^{2}\left|T_{p}^{\alpha}\left(e^{i \theta}\right)\right|^{2}}
$$

where $\alpha \in \mathbb{D}$ and $T_{p}^{\alpha}=\sum_{k=0}^{p} n_{k}(\alpha) n_{k}$. Gabardo (cf. [7]) proves that $\widehat{w_{\alpha}}(k)=$ $c_{k}, k=0,1, \cdots, p$. The next lemma shows that for each $\alpha$ there exists $H=$ $H(\alpha)$ such that the function $w_{\alpha}$ can be obtained from (17).

Lemma 1 Given $\alpha \in \mathbb{D}, w_{\alpha}(\zeta)=f_{H_{\alpha}}(\zeta)$ for $H_{\alpha}$ the constant function defined by

$$
H_{\alpha}(z)=\frac{\bar{\alpha} \overline{n_{p}(\alpha)}}{\overline{m_{p}(\alpha)}}
$$

The proof of this lemma can be found in [10]. 
Functions $w_{\alpha}$ are related to the Burg entropy functional by the following inequality, obtained by Gabardo (cf. [7]).

$$
\frac{1}{2 \pi} \int_{0}^{2 \pi} \log \left[f_{H}\left(e^{i t}\right)\right] \frac{1-|\alpha|^{2}}{\left|1-\alpha e^{-i t}\right|^{2}} d t \leq \frac{1}{2 \pi} \int_{0}^{2 \pi} \log \left[w_{H_{\alpha}}\left(e^{i t}\right)\right] \frac{1-|\alpha|^{2}}{\left|1-\alpha e^{-i t}\right|^{2}} d t
$$

When $\alpha=0$ (19) yields $w_{0}$, the Burg solution. The next theorem shown by Burg [2] is a consequence of (19).

Theorem 2 (Burg). Let $p \in \mathbb{N}$ and $\left\{c_{k}\right\}_{k=0}^{p}$ be the first $(p+1)$ autocorrelations of a second order stationary process $X=\left\{X_{k}\right\}_{k \in \mathbb{Z}}$ then the density $f_{0}$ of $X$ which maximizes Burg's functional $\varepsilon(f)$ restricted to the conditions

$$
\frac{1}{2 \pi} \int_{0}^{2 \pi} e^{-i k t} f\left(e^{i t}\right) d t=c_{k}, \quad k=0, \cdots, p
$$

is

$$
f_{0}\left(e^{i t}\right)=\frac{1}{\left|n_{p}\left(e^{i t}\right)\right|^{2}}, t \in[0,2 \pi] .
$$

Now, we consider a matrix version of the previous inequality. A Hermitian integrable $q \times q$ matrix function $\mathbf{W}$ on $\mathbb{T}$ is called a weight $q \times q$ matrix if $\mathbf{W} \geq \mathbf{0}$ and $\operatorname{det} \mathbf{W}\left(e^{i t}\right) \neq 0$ holds almost everywhere [5]. Let us suppose that $\mathbf{W}$ is a weight $q \times q$ matrix on $\mathbb{T}$. The space $\mathbf{L}^{2}(\mathbf{W})$ is defined by

$$
\mathbf{L}^{2}(\mathbf{W})=\left\{\boldsymbol{\Psi}: \mathbb{T} \rightarrow \mathbb{C}^{q \times q} \mid\|\mathbf{\Psi}\|_{\mathbf{W}}^{2}=\frac{1}{2 \pi} \int_{0}^{2 \pi} \operatorname{Trace}\left(\mathbf{\Psi} \mathbf{W} \mathbf{\Psi}^{*}\right) d t<\infty\right\} .
$$

This space, endowed with the inner product

$$
\langle\boldsymbol{\Phi}, \mathbf{\Psi}\rangle_{\mathbf{W}}=\frac{1}{2 \pi} \int_{0}^{2 \pi} \operatorname{Trace}\left(\mathbf{\Phi} \mathbf{W} \mathbf{\Psi}^{*}\right) d t, \quad \boldsymbol{\Phi}, \mathbf{\Psi} \in \mathbf{L}^{2}(\mathbf{W}),
$$

is a Hilbert space [11].

Following [11] we introduce in $\mathbf{L}^{2}(\mathbf{W})$ a matrix inner product defined by

$$
(\mathbf{\Phi}, \mathbf{\Psi})_{\mathbf{W}}=\frac{1}{2 \pi} \int_{0}^{2 \pi} \mathbf{\Phi} \mathbf{W} \Psi^{*} d t, \quad \boldsymbol{\Phi}, \boldsymbol{\Psi} \in L^{2}(\mathbf{W}) .
$$

We state the following lemma, whose proof is straightforward.

Let $\mathcal{E}_{p}\left(\mathbb{C}^{q \times q)}\right.$ be the manifold spanned by $\left\{e_{k} \mathbf{E}^{i, j}\right\}_{k=0, \ldots, p}^{i, j=1, \ldots, q}$, that is, the set of all matrices whose components are trigonometric polynomials on $\mathbb{T}$ of degree at most $p$.

The following result is analogous to that obtained in(10). 
Proposition 2 Given $\mathbf{P} \in \mathcal{E}_{p}\left(\mathbb{C}^{q \times q}\right)$, we obtain

$$
\left(\left(\mathbf{T}_{p}^{\xi}\right)^{*}, \mathbf{P}^{*}\right)_{\mathbf{F}_{\mathbf{H}}}=\mathbf{P}(\xi)
$$

Proof: From the proposition 1, we obtain

$$
\begin{aligned}
P_{i j}(\xi) & =\left\langle P^{(j)},\left(T_{p}^{\xi}\right)_{i}\right\rangle_{p}=\left\langle\mathbf{P} E^{j}, \mathbf{T}_{p}^{\xi} E^{i}\right\rangle_{p} \\
& =\sum_{l=0}^{p} \sum_{k=0}^{p}\left\langle e_{l} \widehat{\mathbf{P}}(l) E^{j}, e_{k} \widehat{\mathbf{T}_{p}^{\xi}}(k) E^{i}\right\rangle_{p} \\
& =\sum_{l=0}^{p} \sum_{k=0}^{p}\left\langle\mathbf{R}_{k-l} \widehat{\mathbf{P}}(l) E^{j}, \widehat{\mathbf{T}_{p}^{\xi}}(k) E^{i}\right\rangle_{\mathbb{C}^{q}} \\
& =\sum_{l=0}^{p} \sum_{k=0}^{p}\left\langle\frac{1}{2 \pi} \int_{0}^{2 \pi} e^{-i(k-l) t} \mathbf{F}_{\mathbf{H}}\left(e^{i t}\right) d t \widehat{\mathbf{P}}(l) E^{j}, \widehat{\mathbf{T}_{p}^{\xi}}(k) E^{i}\right\rangle_{\mathbb{C}^{q}} \\
& =\left\langle\frac{1}{2 \pi} \int_{0}^{2 \pi} \sum_{k=0}^{p} \widehat{\mathbf{T}_{p}^{\xi}}(k)^{*} e^{-i k t} \mathbf{F}_{\mathbf{H}}\left(e^{i t}\right) \sum_{l=0}^{p} \widehat{\mathbf{P}}(l) e^{i l t} d t E^{j}, E^{i}\right\rangle_{\mathbb{C}^{q}} \\
& =\left\langle\frac{1}{2 \pi} \int_{0}^{2 \pi}\left(\mathbf{T}_{p}^{\xi}\right)^{*}\left(e^{i t}\right) \mathbf{F}_{\mathbf{H}}\left(e^{i t}\right) \mathbf{P}\left(e^{i t}\right) d t E^{j}, E^{i}\right\rangle_{\mathbb{C}^{q}} \\
& =\left\langle\left(\left(\mathbf{T}_{p}^{\xi}\right)^{*}, \mathbf{P}^{*}\right)_{\mathbf{F}_{\mathbf{H}}} E^{j}, E^{i}\right\rangle_{\mathbb{C}^{q}} \cdot \mathbf{\square}
\end{aligned}
$$

In the next corollary, we show that the matrix $\mathbf{T}_{p}^{z}(z)$ is positive definite.

Corollary 1 For each $\alpha \in \mathbb{D}$,

$$
\mathbf{T}_{p}^{\alpha}(\alpha)>\mathbf{0}
$$

Proof: This result is a direct consequence of proposition 2. In fact,

$$
\mathbf{T}_{p}^{\alpha}(\alpha)=\left(\left(\mathbf{T}_{p}^{\alpha}\right)^{*},\left(\mathbf{T}_{p}^{\alpha}\right)^{*}\right)_{\mathbf{F}_{\mathbf{H}}}=\frac{1}{2 \pi} \int_{0}^{2 \pi}\left(\mathbf{T}_{p}^{\alpha}\right)^{*}\left(e^{i t}\right) \mathbf{F}_{\mathbf{H}}\left(e^{i t}\right) \mathbf{T}_{p}^{\alpha}\left(e^{i t}\right)>\mathbf{0}
$$

because $\mathbf{F}_{\mathbf{H}}>\mathbf{0}$.

Lemma 2 For each $\alpha \in \mathbb{D}$, let $\mathbf{H}_{\alpha}(z)=\bar{\alpha} \mathbf{N}_{p}^{*}(\alpha)\left[\mathbf{M}_{p}^{*}\right]^{-1}(\alpha)$. Then,

$$
\mathbf{H}_{\alpha}^{*} \mathbf{H}_{\alpha}<\mathbf{I}_{q \times q} .
$$


Proof: From the Christoffel-Darboux formula and corollary 1 we have

$$
\mathbf{0}<\mathbf{T}_{p}^{\alpha}(\alpha)=\frac{\mathbf{M}_{p}(\alpha) \mathbf{M}_{p}^{*}(\alpha)-|\alpha|^{2} \mathbf{N}_{p}(\alpha) \mathbf{N}_{p}^{*}(\alpha)}{1-|\alpha|^{2}} .
$$

That is, for each non null $x \in \mathbb{C}^{q}$

$$
x^{*}\left(\mathbf{M}_{p}(\alpha) \mathbf{M}_{p}^{*}(\alpha)-|\alpha|^{2} \mathbf{N}_{p}(\alpha) \mathbf{N}_{p}^{*}(\alpha)\right) x>\mathbf{0} .
$$

We can rewrite the previous inequality as,

$$
x^{*} \mathbf{M}_{p}(\alpha)\left(\mathbf{I}_{q \times q}-\alpha \mathbf{M}_{p}(\alpha)^{-1} \mathbf{N}_{p}(\alpha) \bar{\alpha} \mathbf{N}_{p}^{*}(\alpha)\left(\mathbf{M}_{p}^{*}(\alpha)\right)^{-1}\right) \mathbf{M}_{p}^{*}(\alpha) x>\mathbf{0} .
$$

(From [9] we know that $\mathbf{M}_{p}(\alpha)^{-1}$ exists).

Thus,

$$
u^{*}\left(\mathbf{I}_{q \times q}-\mathbf{H}_{\alpha}^{*} \mathbf{H}_{\alpha}\right) u>\mathbf{0},
$$

where $u=\mathbf{M}_{p}^{*} x$.

Proposition 3 For each $\alpha \in \mathbb{D}$, $\mathbf{T}_{p}^{\alpha}$ is invertible. Furthermore, this inverse is given by

$$
\left[\mathbf{T}_{p}^{\alpha}\right]^{-1}=\left[\mathbf{M}_{p}^{*}\right]^{-1}(\alpha)\left[\mathbf{N}_{p}^{-1} \mathbf{M}_{p}-e^{i t} \mathbf{H}_{\alpha}\right]^{-1} \mathbf{N}_{p}^{-1}\left(1-\bar{\alpha} e^{i t}\right) .
$$

Proof: From [9], we know that $\left[\mathbf{N}_{p}^{-1} \mathbf{M}_{p}-e^{i t} \mathbf{H}_{\alpha}\right]^{-1}$ exists. Therefore,

$$
\begin{aligned}
& {\left[\mathbf{M}_{p}^{*}\right]^{-1}(\alpha)\left[\mathbf{N}_{p}^{-1} \mathbf{M}_{p}-e^{i t} \mathbf{H}_{\alpha}\right]^{-1} \mathbf{N}_{p}^{-1}} \\
& \quad=\left[\mathbf{N}_{p}\left(\mathbf{N}_{p}^{-1} \mathbf{M}_{p}-e^{i t} \mathbf{H}_{\alpha}\right) \mathbf{M}_{p}^{*}(\alpha)\right]^{-1} \\
& \quad=\left[\mathbf{M}_{p} \mathbf{M}_{p}^{*}(\alpha)-e^{i t} \mathbf{N}_{p} \bar{\alpha} \mathbf{N}_{p}^{*}(\alpha)\left(\mathbf{M}_{p}^{*}\right)^{-1}(\alpha) \mathbf{M}_{p}^{*}(\alpha)\right]^{-1} \\
& \quad=\left[\mathbf{M}_{p} \mathbf{M}_{p}^{*}(\alpha)-e^{i t} \mathbf{N}_{p} \bar{\alpha} \mathbf{N}_{p}^{*}(\alpha)\right]^{-1} .
\end{aligned}
$$

For each $\alpha \in \mathbb{D}$, we define the function

$$
\mathbf{W}_{\alpha}\left(e^{i t}\right)=\frac{1-|\alpha|^{2}}{\left|e^{i t}-\alpha\right|^{2}}\left[\mathbf{T}_{p}^{\alpha}\left(e^{i t}\right)^{*}\right]^{-1}\left[\mathbf{T}_{p}^{\alpha}(\alpha)\right]\left[\mathbf{T}_{p}^{\alpha}\left(e^{i t}\right)\right]^{-1}
$$

$\mathbf{W}_{\alpha}$ represents the matrix version of (18).

The following proposition shows that the matrix function $\mathbf{W}_{\alpha}$ can be obtained from (15) for some $\mathbf{H}_{\alpha}$ and therefore $\widehat{\mathbf{W}_{\alpha}}(k)=\mathbf{R}_{k}, k=0,1, \cdots, p$. 
Proposition 4 Given $\alpha \in \mathbb{D}$, the functions $\mathbf{W}_{\alpha}$ can be obtained from (15), setting $\mathbf{H}$ to be the constant matrix

$$
\mathbf{H}_{\alpha}(z)=\bar{\alpha} \mathbf{N}_{p}^{*}(\alpha)\left[\mathbf{M}_{p}^{*}\right]^{-1}(\alpha) .
$$

Proof: Let $\alpha \in \mathbb{D}$. From proposition 3, we obtain

$$
\left[\mathbf{T}_{p}^{\alpha}\right]^{-1}=\left[\mathbf{M}_{p}^{*}\right]^{-1}(\alpha)\left[\mathbf{N}_{p}^{-1} \mathbf{M}_{p}-e^{i t} \mathbf{H}_{\alpha}\right]^{-1} \mathbf{N}_{p}^{-1}\left(1-\bar{\alpha} e^{i t}\right) .
$$

Furthermore, from the Christoffel-Darboux formula,

$$
\begin{aligned}
\mathbf{T}_{p}^{\alpha}(\alpha) & =\frac{\mathbf{M}_{p}(\alpha) \mathbf{M}_{p}^{*}(\alpha)-|\alpha|^{2} \mathbf{N}_{p}(\alpha) \mathbf{N}_{p}^{*}(\alpha)}{1-|\alpha|^{2}} \\
& =\mathbf{M}_{p}(\alpha) \frac{\mathbf{I}_{q \times q}-|\alpha|^{2} \mathbf{M}_{p}^{-1}(\alpha) \mathbf{N}_{p}(\alpha) \mathbf{N}_{p}^{*}(\alpha)\left[\mathbf{M}_{p}^{*}(\alpha)\right]^{-1}}{1-|\alpha|^{2}} \mathbf{M}_{p}^{*}(\alpha) \\
& =\mathbf{M}_{p}(\alpha) \frac{\mathbf{I}_{q \times q}-\mathbf{H}_{\alpha}^{*} \mathbf{H}_{\alpha}}{1-|\alpha|^{2}} \mathbf{M}_{p}^{*}(\alpha) .
\end{aligned}
$$

Therefore,

$$
\begin{aligned}
\mathbf{W}_{\alpha}\left(e^{i t}\right) & =\frac{1-|\alpha|^{2}}{\left|e^{i t}-\alpha\right|^{2}}\left[\mathbf{T}_{p}^{\alpha}\left(e^{i t}\right)^{*}\right]^{-1}\left[\mathbf{T}_{p}^{\alpha}(\alpha)\right]\left[\mathbf{T}_{p}^{\alpha}\left(e^{i t}\right)\right]^{-1} \\
& =\frac{1-|\alpha|^{2}}{\left|e^{i t}-\alpha\right|^{2}}\left(1-\alpha e^{-i t}\right)\left[\mathbf{N}_{p}^{*}\right]^{-1}\left\{\left[\mathbf{N}_{p}^{-1} \mathbf{M}_{p}-e^{i t} \mathbf{H}_{\alpha}\right]^{*}\right\}^{-1} \mathbf{M}_{p}^{-1}(\alpha) \\
& \times \mathbf{M}_{p}(\alpha) \frac{\mathbf{I}_{q \times q}-\mathbf{H}_{\alpha}^{*} \mathbf{H}_{\alpha}}{1-|\alpha|^{2}} \mathbf{M}_{p}^{*}(\alpha) \\
& \times\left[\mathbf{M}_{p}^{*}\right]^{-1}(\alpha)\left[\mathbf{N}_{p}^{-1} \mathbf{M}_{p}-e^{i t} \mathbf{H}_{\alpha}\right]^{-1} \mathbf{N}_{p}^{-1}\left(1-\bar{\alpha} e^{i t}\right) \\
& =\left[\mathbf{N}_{p}^{*}\right]^{-1}\left\{\left[\mathbf{N}_{p}^{-1} \mathbf{M}_{p}-e^{i t} \mathbf{H}_{\alpha}\right]^{*}\right\}^{-1}\left(\mathbf{I}_{q \times q}-\mathbf{H}_{\alpha}^{*} \mathbf{H}_{\alpha}\right) \\
& \times\left[\mathbf{N}_{p}^{-1} \mathbf{M}_{p}-e^{i t} \mathbf{H}_{\alpha}\right]^{-1} \mathbf{N}_{p}^{-1} \\
& =\mathbf{F}_{\mathbf{H}_{\alpha}} . \mathbf{-}
\end{aligned}
$$

In the next theorem, we use the Krein functional to generalize inequality (19) obtained by Gabardo.

Proposition 5 The following inequality is valid:

$\frac{1}{2 \pi} \int_{0}^{2 \pi} \log \operatorname{det}\left[\mathbf{F}_{\mathbf{H}}\left(e^{i t}\right)\right] \frac{1-|\alpha|^{2}}{\left|1-\alpha e^{-i t}\right|^{2}} d t \leq \frac{1}{2 \pi} \int_{0}^{2 \pi} \log \operatorname{det}\left[\mathbf{W}_{\alpha}\left(e^{i t}\right)\right] \frac{1-|\alpha|^{2}}{\left|1-\alpha e^{-i t}\right|^{2}} d t$.

Rev.Mate.Teor.Aplic. (ISSN print: 1409-2433; online: 2215-3373) Vol. 24(1): 97-113, January 2017 
Proof: Using Jensen's inequality (see [5]) and proposition 2, we obtain

$$
\begin{aligned}
\frac{1}{2 \pi} & \int_{0}^{2 \pi} \log \operatorname{det}\left(\mathbf{F}_{\mathbf{H}} \mathbf{W}_{\alpha}^{-1}\right) \frac{1-|\alpha|^{2}}{\left|e^{i t}-\alpha\right|^{2}} d t \\
& =\frac{1}{2 \pi} \int_{0}^{2 \pi} \log \operatorname{det}\left(\mathbf{F}_{\mathbf{H}} \frac{\left|e^{i t}-\alpha\right|^{2}}{1-|\alpha|^{2}} \mathbf{T}_{p}^{\alpha}\left(e^{i t}\right)^{*}\left[\mathbf{T}_{p}^{\alpha}(\alpha)\right]^{-1} \mathbf{T}_{p}^{\alpha}\left(e^{i t}\right)\right) \frac{1-|\alpha|^{2}}{\left|e^{i t}-\alpha\right|^{2}} d t \\
& =\frac{1}{2 \pi} \int_{0}^{2 \pi} \log \operatorname{det}\left(\frac{\left|e^{i t}-\alpha\right|^{2}}{1-|\alpha|^{2}} \mathbf{T}_{p}^{\alpha}\left(e^{i t}\right)^{*} \mathbf{F}_{\mathbf{H}} \mathbf{T}_{p}^{\alpha}\left(e^{i t}\right)\left[\mathbf{T}_{p}^{\alpha}(\alpha)\right]^{-1}\right) \frac{1-|\alpha|^{2}}{\left|e^{i t}-\alpha\right|^{2}} d t \\
& \leq \log \operatorname{det} \frac{1}{2 \pi} \int_{0}^{2 \pi}\left(\frac{1-|\alpha|^{2}}{\left|e^{i t}-\alpha\right|^{2}} \mathbf{T}_{p}^{\alpha}\left(e^{i t}\right)^{*} \mathbf{F}_{\mathbf{H}} \mathbf{T}_{p}^{\alpha}\left(e^{i t}\right)\left[\mathbf{T}_{p}^{\alpha}(\alpha)\right]^{-1}\right) \frac{\left|e^{i t}-\alpha\right|^{2}}{1-|\alpha|^{2}} d t \\
& =\log \operatorname{det} \frac{1}{2 \pi} \int_{0}^{2 \pi} \mathbf{T}_{p}^{\alpha}\left(e^{i t}\right)^{*} \mathbf{F}_{\mathbf{H}} \mathbf{T}_{p}^{\alpha}\left(e^{i t}\right) d t\left[\mathbf{T}_{p}^{\alpha}(\alpha)\right]^{-1} \\
& =\log \operatorname{det}\left(\left(\mathbf{T}_{p}^{\alpha}\right)^{*},\left(\mathbf{T}_{p}^{\alpha}\right)^{*}\right)_{\mathbf{F}_{\mathbf{H}}}\left[\mathbf{E}_{p}^{\alpha}(\alpha)\right]^{-1} \\
& =\log \operatorname{det} \mathbf{T}_{p}^{\alpha}(\alpha)\left[\mathbf{T}_{p}^{\alpha}(\alpha)\right]^{-1}=0 . \mathbf{-}
\end{aligned}
$$

Gabardo [7] points out that Dym in [6] presents an analogous matrix version of the preceding inequality, but for the Wiener class.

If we choose $\alpha=0$, we obtain from the previous theorem yields that the matrix function $\mathbf{W}_{\alpha}$ maximizes the Burg functional.

Theorem 3 Given a strictly positive definite sequence $\left\{\mathbf{R}_{k}\right\}_{k=-p}^{p}$ and a $q \times$ $q$ hermitian non negative matrix measure $\Omega$ on $\mathbb{T}$, absolutely continuous with respect to the Lebesgue measure on $\mathbb{T}$, with density $\mathbf{F}>0$, the maximum of $\varepsilon(\mathbf{F})$ constrained to the restrictions

$$
\frac{1}{2 \pi} \int_{0}^{2 \pi} e^{-i k t} \mathbf{F}\left(e^{i t}\right) d t=\mathbf{R}_{k}, k=-p, \cdots, p
$$

is attained at

$$
\mathbf{F}_{0}\left(e^{i t}\right)=\left(\mathbf{M}_{p}^{*}\right)^{-1}\left(e^{i t}\right) \mathbf{M}_{p}^{-1}\left(e^{i t}\right)
$$

\section{Conclusions}

In this paper we consider the Krein functional $\epsilon(\mathbf{W} ; \alpha)$ and show that there exists a family of matrix functions $\left\{\mathbf{W}_{\alpha}\right\}$ that verifies the matrix version of (1), in such a way that the inequality $\epsilon(\mathbf{F}, \alpha) \leq \epsilon\left(\mathbf{W}_{\alpha}, \alpha\right)$ is valid for every matrix function $\mathbf{F}$ which is integrable on the unit circle satisfying the same restrictions as $\mathbf{W}_{\alpha}$. In particular, when $\alpha=0$ yields $W_{0}$, obtain the Burg solution. 


\section{References}

[1] Arov, D.Z.; Krein, M.G. (1981) "Problem of search of the minimun of entropy in indeterminate extension problems", Funt. Anal. Appl. 15(2): 123-126.

[2] Burg, J.P. (1975) Maximum Entropy Spectral Analysis. Ph.D. Dissertation, Stanford University, Stanford, California.

[3] Choi, B.S. (1986) "On the relation bemtween the maximum entropy probability density function and the autoregressive model", IEEE Trans. Acoust. Speech Signal Process. ASSP-34.

[4] Choi, B.S. (1993) "Multivariate maximum entropy spectrum", J. Multivariate Anal. 46(1): 56-60.

[5] Delsarte, P.; Genin, Y.; Kamp, Y. (1978) "Orthogonal polynomial matrices on the unit circles", IEEE Trans. Circuits Syst. 25(3): 149-160.

[6] Dym, H. (1989) "J. contractive matrix function, reproducing kernel Hilbert spaces and interpolation", CBMS Regional Conference Series in Mathematics 71, A.M.S., Providence, RI.

[7] Gabardo, J.P. (1993) "Extension of positive definite distributions and maximum entropy”, Mem. Am. Math. Soc., Providence RI, 102(489).

[8] Landau, H.J. (1987) "Maximum entropy and the moment problem", Bull. Am. Math. Soc. 16(1): 47-77.

[9] Marcano, J.G.; Moran, M.D. (2003) “The Arov-Grossman model and Burg multivariate entropy" J. Fourier Anal. Appl. 9(6): 623-647.

[10] Marcano, J.G.; Moran, M.D. (2005) "The Arov-Grossman model and Burg's entropy”, in: R. Baez-Yates, J. Glaz, H. Gzyl, J. Hüsler \& J.L. Palacios (Eds.) Recent Adv. Appl. Probab., Springer, Boston: 329-364.

[11] Masani, P. (1966) Recent trends in multivariate prediction theory, in: P.R. Krishnaiah (Ed.) Multivariate Analysis, Academic Press, New York: 351382. 
